\title{
POUR UNE SPIRITUALITÉ DU LOISIR ${ }^{1}$
}

\author{
Gervais DESCHÊNES
}

Université du Québec à Chicoutimi

"Pendant que Dieu joue, le monde se fait.» - Wilhelm Gottfried Leibniz

\section{Introduction}

Toute tentative de définir le loisir se heurte à un obstacle de taille : l'évolution rapide de ce phénomène. Pour certains théoriciens, le loisir est simplement synonyme de temps libre. D'aucuns l'utilisent pour se référer aux activités spécifiques d'une programmation. D'autres encore emploient ce terme pour décrire l'état d'une expérience positive, généralement caractérisée par des sentiments de liberté et de motivations intrinsèques. La disparité de sens découle du fait que le loisir touche à plusieurs réalités de la vie quotidienne. Pour sa part, Lanfant (1972) rattache les problèmes méthodologiques d'une définition accordée au loisir à l'imprécision et à l'écart entre les langages populaires et scientifiques, aux jugements critiques de l'approche subjective et, finalement, à la prolifération idéologique dans l'interprétation des données empiriques du loisir. Les nombreux débats théoriques et idéologiques entre sociologues, psychologues et philosophes témoignent de la difficulté de faire consensus sur la définition du loisir. Pour notre part, nous choisissons de privilégier une approche anthropologique et religieuse du loisir qui pose la question du sens. Or, dans la société sécularisée actuelle, on peut se demander s'il est possible d'établir des liens entre le loisir et la religion, car pour Dumont (1987, p. 218) : «Le mot religion a subi une telle dérive de sens que les analyses que l'on poursuit sous son étiquette risquent de s'égarer». C'est pourquoi cette étude du loisir s'appuie sur le sens du mot «religion» présenté par Tillich, qui comprend ce concept d'une manière ouverte : 
Être religieux signifie s'interroger passionnément sur le sens de notre vie et être ouvert aux réponses, même si elles nous ébranlent en profondeur. Une telle conception fait de la religion quelque chose d'universellement humain, encore qu'elle s'écarte de ce qu' on entend ordinairement par religion. Celleci, en tant que dimension de la profondeur, n'est pas la foi à l'existence des dieux, ni même à l'existence d'un seul dieu. Elle ne consiste pas en attitudes et en organisations où se manifeste le lien de l'homme avec son dieu. Nul ne contestera que les religions historiques soient «religion» en ce sens. Mais une religion en sa véritable essence est plus que cela : elle s'identifie à l'être de l'homme pour tout ce qui met en cause le sens de la vie comme son existence même (Tillich, 1969, p. 49).

Dans cet article, nous allons explorer le monde de la sociologie du loisir, des sciences humaines et de la théologie. Ainsi, il s'agira de comprendre la pratique du loisir à l'aide du modèle théorique élaboré par John R. Kelly dans son livre Freedom to $\mathrm{Be}^{2}$. Cette construction théorique représente une étude holistique du loisir considérant celui-ci comme un processus complexe et évolutif. Ce nouveau mouvement accorde de l'importance à la dimension historique et structurelle du loisir qui est conditionnée par la culture, l'ethnie, le sexe, les classes sociales, la famille, la religion, etc. ${ }^{3}$. Pour Kelly, le loisir est une expérience subjective qui se traduit dans l'expression de soi, la créativité, l'apprentissage et la croissance de l'humain. Essayons d'expliquer brièvement en quoi consiste le modèle de Kelly.

\section{Définition du modèle théorique de Kelly}

Kelly s'appuie sur différents penseurs (théologiens, philosophes, sociologues, psychologues, etc.) pour réaliser une construction théorique du loisir. Son modèle théorique vise à reconnaître le loisir comme une dimension fondamentale du processus de la vie. Selon lui, l'élaboration de ce modèle n'est jamais terminée, mais demeure ouverte à l'évolution des humains et de la société dans un continuel changement; l'humain est toujours engagé dans une manière de devenir. C'est pourquoi il ne convient pas d'aborder ce modèle comme un absolu. Kelly ne fait pas de son système une dogmatique, car pour lui « [...] il n'y a pas finalement de « vérités » ou de réalités absolues, mais seulement le travail réflexif d'une explication qui cherche à être systématique et ouverte à la correction si possible. La théorie n'est pas alors un théorème prouvé à tout moment, mais un processus de réflexion pour une explication » (Kelly, 1997, p. 404).

Pour une théorie explicative du loisir, Kelly adopte à la fois les approches sociale et existentielle. L'approche sociale implique que les forces sociales déterminent le comportement de l'humain, tandis que l'approche existentielle suppose que l'humain est appelé à assumer sa liberté. En tant qu'acteur social, il prend le risque de la décision menant à l'action. Ainsi, les approches sociale et existentielle du modèle kellien reposent sur le principe suivant lequel la réalité des forces sociales et le risque de la décision font tous deux partie de la vie humaine. 
Pour Kelly, le loisir n'est pas un simple concept ni un phénomène arrêté, mais plutôt une réalité complexe, plurielle, ambivalente, contingente aux nombreuses facettes dans le processus de l'existence de l'humain, qui nous oblige à le comprendre comme un «état de devenir». Ainsi, le loisir a un caractère orienté vers l'avenir et cette orientation le définit mieux que le temps, le lieu, la forme ou même le résultat. Par conséquent, le modèle théorique de Kelly est une dynamique toujours ouverte au perfectionnement.

Ce modèle s'articule, en outre autour d'une spirale composée de huit théories sous forme de métaphores. Kelly entend expliquer le loisir en disant et en présumant que cette pratique est «quelque chose comme ceci» (Kelly, 1987, p. 3). Autrement dit, la théorie vue comme métaphore suggère que «si vous voyez quelque chose de cette perspective, il peut être bien compris de cette manière » (Kelly, 1987, p. 1). Ainsi, la métaphore est une manière de saisir la réalité du loisir et de lui donner un ensemble d'angles explicatifs pour mieux le comprendre. En même temps, les métaphores s'intègrent les unes aux autres de façon dialectique. En fait, ce modèle théorique est basé sur une double dialectique.

Pour le premier type, Kelly s'inspire de la pensée dialectique hégélienne, «thèse-antithèse-synthèse ». Ainsi, dans chaque métaphore, il nous présente sa thèse qu'il développe. Toutefois, il se rend compte que sa thèse n'épuise pas toute la réalité du loisir et qu'elle se trouve contredite par des réalités qu'il nomme dans une antithèse ; ce qui l' amène à développer une deuxième métaphore qui comprend elle-même une thèse et une antithèse menant, elle aussi, à une troisième métaphore. Cette série de métaphores constitue le deuxième type de dialectique faisant référence à la philosophie heideggerienne qui développe l'image de la spirale herméneutique; celle-ci propose un modèle d'interprétation où la somme des parties constitue la totalité. Chaque métaphore est interprétée dans une perspective de la totalité qui, à son tour, est reconstruite par l'inclusion de nouvelles métaphores; ce processus de la spirale herméneutique est sans fin. Ainsi, chaque métaphore est contre-argumentée, corrigée mais non rejetée afin de poursuivre l'analyse théorique du loisir dans la métaphore suivante du trajet dialectique de la spirale. Pour notre propos, il nous paraît utile de présenter ici le schéma du modèle théorique de Kelly.

Kelly explique comment l'humain peut trouver son identité profonde dans le loisir (métaphore 1, 2, 3 et 4), mais aussi s'insérer dans la société (métaphore 5,6 et 7) pour déboucher sur l'essence même de son humanité (métaphore 8). Reprenons brièvement chacune des métaphores qui composent ce processus d'humanisation. La première est l' «expérience immédiate» qui se comprend comme un «état d'esprit» (métaphore 1). Pris dans son acception «existentielle», le loisir, lors des prises de décision qu'il implique, oriente l'action qui donne un sens à la vie (métaphore 2). À plus long terme, le loisir s'inscrit dans une dynamique 
FIGURE 1

Modèle théorique du loisir de Kelly

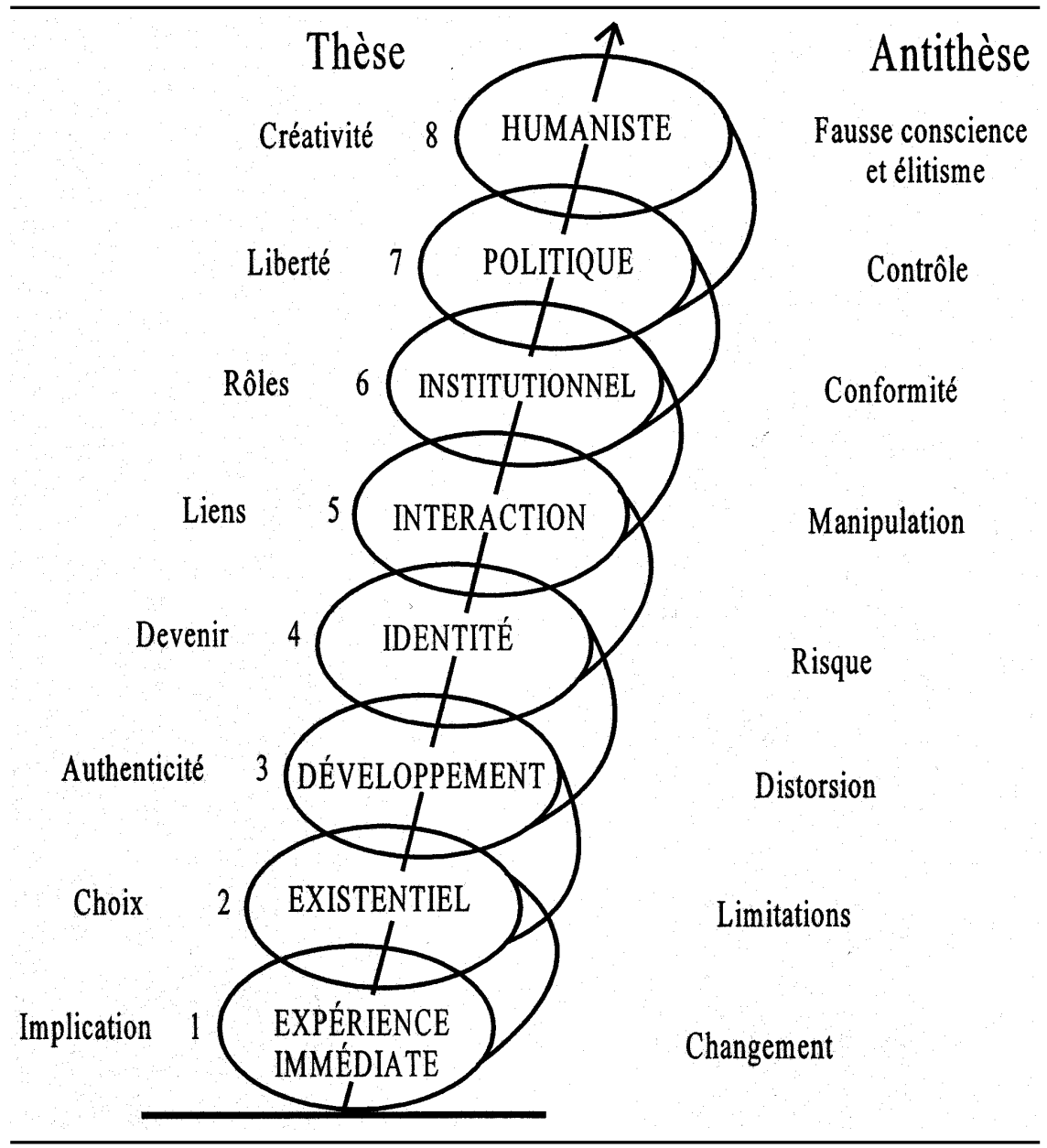

de «développement de soi » dans tout le parcours de vie de l'humain (métaphore 3). Dans la quête de «l'identité » personnelle et sociale, il crée le caractère intime des relations humaines stimulant ainsi la création d'une communauté (métaphore 4). Le loisir débouche sur des « interactions sociales » qui nous apprennent, par la rencontre, où et qui nous sommes dans le système social (métaphore 5). Le loisir acquiert alors sa dimension «institutionnelle » étant donné que la famille, le travail, la religion et la culture intègrent cette valeur (métaphore 6). Dans les sociétés stratifiées, le loisir se révèle un mécanisme d'aliénation en tant qu'instrument de contrôle social à caractère mercantile et fétichiste, et, en ce sens, il devient une 
réalité éminemment «politique» (métaphore 7). Finalement, le loisir peut jouer le rôle d'agent libérateur favorisant l'action créatrice du «pas encore» rendant possible la réalisation de soi, de la culture et du monde ; il devient alors un facteur d'«humanisme» (métaphore 8).

Kelly nous aura transmis que le loisir n'est pas un temps perdu. Bien au contraire : il est associé aux notions, à la fois d'aliénation, de liberté et de créativité au cœur de l'aventure de chaque humain. En réalité, il en fait un acte fondamental d'ouverture, d'autonomie et de restitution de l'humain. Donc, dans le loisir créatif, l'humanisation se produit dans un milieu de loisir qui permet de faire des découvertes et de construire la communauté. Dans cette perspective, le loisir est éminemment « sérieux » et fondamental; ce qui s'y joue, c'est l'essence même de l'humanité. Il est donc fondamental pour l'humain d'aujourd'hui d'apprendre à assumer son identité radicale d' «être de loisir».

Dans la métaphore humaniste, Kelly affirme que l'humain se réalise dans la créativité (métaphore 8). D'une certaine façon, l'acte de création est de faire advenir un monde. Pour Kelly, le loisir est une forme d'expérience religieuse (métaphore 6) et, en ce sens, il peut s'identifier à une forme de religion, mais Kelly n'évoque pas explicitement la relation du sacré et de la transcendance dans la pratique du loisir.

Suite au développement des huit métaphores, Kelly soutient lui-même qu'il est envisageable d'explorer la possibilité de créer d'autres métaphores qui peuvent saisir à nouveau l'ensemble de son modèle (Kelly, 1987, p. 18). Ainsi, dans la perspective d'une théorie ouverte, nous proposons d'ajouter deux autres métaphores à son modèle. Dans la neuvième métaphore, à partir des sciences humaines, nous élaborons une anthropologie du sacré (Durkheim, 1912; Otto) et montrons ainsi la dimension religieuse du loisir. Par la suite, dans la dixième métaphore, nous montrons que les réalités fondamentales du christianisme (Rahner et Moltmann) peuvent être lues dans une perspective de loisir et de jeu ${ }^{4}$. Voici le modèle théorique enrichi du loisir de Kelly bonifié des métaphores du «religieux » et de la «transcendance $»^{5}$.

\section{La métaphore « religieuse»}

Nous proposons ici une neuvième métaphore, soit celle du « religieux », où apparaît l'aspect sacré6 du loisir. À ce sujet, il nous semble que les spécialistes du loisir devraient réfléchir davantage sur la question de la présence du sacré dans le monde et sur la possibilité de sa manifestation dans le loisir tout en respectant, bien sûr, les croyances de chacun. Ainsi, la thèse de cette métaphore traduit la reconnaissance de la manifestation du sacré, en particulier, dans le loisir. Voici donc une description sommaire de cette nouvelle métaphore sur la dimension religieuse du loisir. 
FIGURE 2

Modèle théorique enrichi du loisir de Kelly

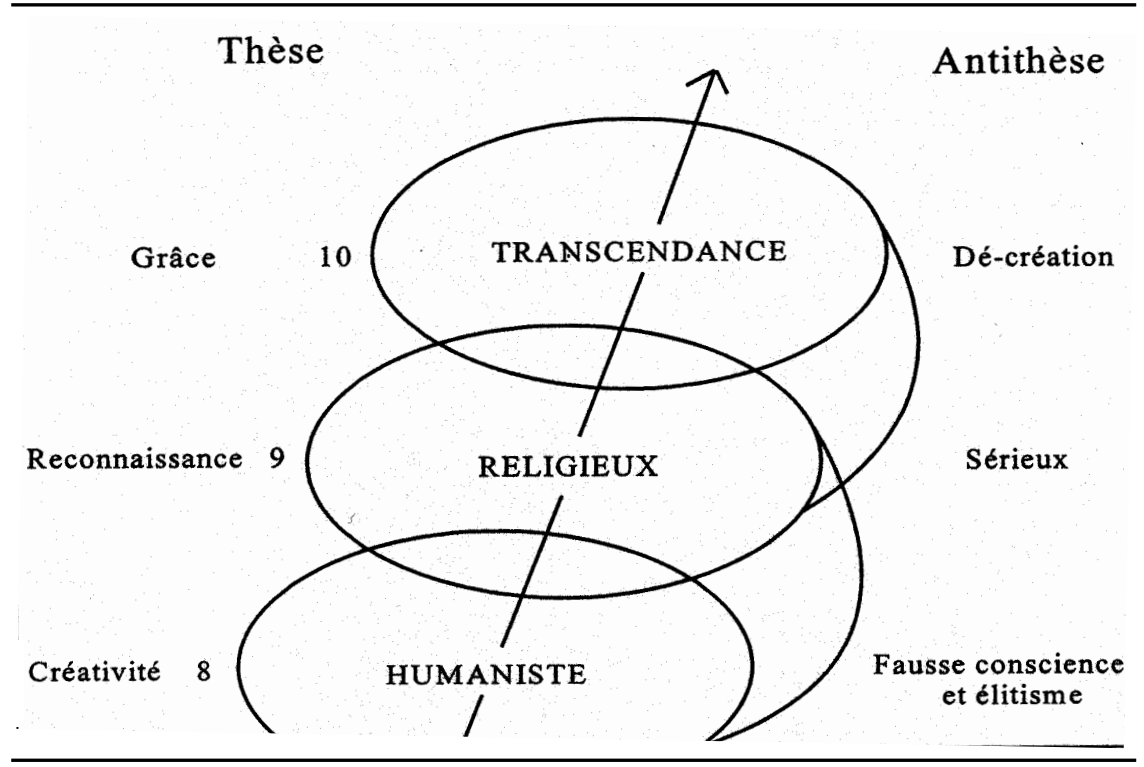

La survivance du sacré en contexte de post-modernité est une réalité et les rites religieux traditionnels de la chrétienté n'expriment plus exclusivement l'expérience du sacré. D'ailleurs, de plus en plus de sociologues montrent la dimension sacrale du loisir, et, plus particulièrement, dans les manifestations sportives. Pour bien comprendre ce lien nouveau, il faut revenir aux théories explicatives du sacré de Durkheim et de Otto en vue de découvrir davantage cette manifestation du sacré dans le loisir qui devient alors en quelque sorte un phénomène rituel. Une mythologie comme jeu sacré est alors envisageable pour une anthropologie de l'humain dans la post-modernité. La métaphore est contre-argumentée par l'antithèse de la lourdeur du sérieux du sacré.

Dans la société dite «de chrétienté », le sacré était le monopole de l'Église et il était identifié aux rites religieux. Or, en contexte de postmodernité, ces rites religieux traditionnels de la chrétienté n'expriment plus exclusivement l'expérience du sacré et cette dernière, se métamorphose ${ }^{7}$. On assiste alors à un changement radical de la compréhension du monde. On se déplace du monde agraire vers le monde industriel et l'humain de la postmodernité se détache progressivement d'une vision passéiste et fixiste vers un monde en pleine mutation. En outre, la postmodernité se caractérise par le phénomène social de la sécularisation qui est «le processus par lequel des secteurs de la société et de la culture sont soustraits à l'autorité des institutions et des symboles religieux » (Berger, 1971, p. 174). 
D'aucuns pensent que la société sécularisée a pour fondement la pensée positiviste et rationnelle qui refoule la religion dans le domaine du privé. On ne peut plus définir le monde autour de vérités religieuses absolues qui révélaient magiquement un Dieu lointain séparé de la réalité expérientielle de l'humain. Déjà, à l' aube du XX $X^{\mathrm{e}}$ siècle, le philosophe Friedrich Nietzsche, vif critique de la modernité, annonçait une vision prophétique : «Ce que je raconte, c'est l'histoire des deux prochains siècles. Je décris ce qui viendra, ce qui ne peut manquer de venir, l'avènement du nihilisme» (cité dans Granier, 1985, p. 32). Nietzsche venait de proclamer la «mort de Dieu», assassiné par l'humain, qui dans le tréfonds de sa conscience, perd alors le fondement de sa réalité existentielle en l'Être et de ses propres valeurs morales et religieuses. Nietzsche illustre un mouvement métaphysique déterminant dans le mouvement dynamique de la modernité vers la postmodernité. La vérité s'éclipse avec Dieu. C'est alors que l'humain, au prise avec l'angoisse du néant, est voué à créer son propre avenir, sa propre volonté de transcendance qui s'inscrit, au dire de Nietzsche, dans la conquête du droit de créer de nouvelles valeurs.

L'ère de la postmodernité se caractérise entre autres par l'avancement technologique depuis le début du $\mathrm{XX}^{\mathrm{e}}$ siècle qui a créé un changement phénoménal dans l'accroissement de l'information disponible et des interactions personnelles. À titre d'exemple, les inventions de l'automobile, de l'avion, de la radio, de la télévision, des ordinateurs et de la télécommunication ont créé «[...] un déluge de stimulations sociales. Les petites et anciennes communautés, caractérisées par les relations de couple plus stables et constantes, ont été remplacées par des relations vastes et complexes » (Gergen, 1991, p. xi). Cet accroissement de stimulations sociales nous a menés à un «relativisme effréné » à travers toutes les sphères de la vie. Toutes les croyances et les valeurs sont remises en question où les relations humaines peuvent se vivre de manière chaotique de sorte que «le monde moderne nous a menés dans une conscience de systèmes de croyances multiples et conflictuelles » (Godbey, 1997). La postmodernité se caractérise également par le culte de l'individualisme et une société narcissique qui, d'après Lipovetski (1983), n'est plus définie par le désespoir ni même par le sentiment d'absurdité, mais bien par la frivolité et la futilité de l'hédonisme dont le loisir se révèle souvent l'un des portedrapeaux. Ces phénomènes du nihilisme, du culte de l'individualisme et des conflits de valeurs soulèvent des questions. Ainsi, si plus rien n'existe comme absolu, si le nihilisme rejette toute valeur, alors comment pouvons-nous toujours croire à la présence du sacré dans la postmodernité ? Assistons-nous passivement à son évanescence?

Toutefois, dans le monde de la sociologie religieuse, l'existence du sacré constitue un objet d'étude bien réel (Caillois, 1950 ; Eliade, 1965; Tessier, 1991). Le sacré peut être, nous semble-t-il, une force qui prend toujours forme au cœur de la réalité humaine. Eliade (1965, p. 31) montre que chez les primitifs, «le sacré est le réel par excellence, à la fois puissance, efficience, source de vie et de 
fécondité». Cependant, dans les temps modernes, il prétend, comme d'autres observateurs, que le sacré a perdu cette force et ne survit que de façon parcellaire. On assiste alors à la «fragmentation du sacré». Chacun de nous entretient à sa manière son univers sacré : «Au fond, tout se passe comme s'il suffisait pour rendre sacré quelque objet, quelque cause ou quelque être de la tenir pour une fin suprême et de lui consacrer sa vie, c'est-à-dire de lui vouer son temps et ses forces, ses intérêts et ses ambitions, de lui sacrifier au besoin son existence » (Caillois, 1950, p. 179). Afin de mieux comprendre le loisir comme manifestation du sacré, nous avons recours principalement à deux théoriciens fondateurs du concept de sacré : Durkheim (1858-1917) en sociologie et Otto (1860-1937) en phénoménologie religieuse.

\section{Application de la théorie du sacré de Durkheim}

Durkheim considère la manifestation du sacré comme une chose; nous sommes en plein positivisme scientifique. Pour lui, le sacré est d'origine sociale. C'est la société qui se donne un fondement transcendant : la société constitue l'âme du sacré religieux ${ }^{8}$. En d'autres termes, c'est la société qui crée et célèbre ses dieux, pour faire du sacré l'apothéose de la société. Bref, la compréhension du sacré chez Durkheim s'explique par la société. Or, si la société fabrique des dieux, il ne faut pas oublier qu'elle est elle-même constituée par des humains, qui sont la source même de la création de la société, donc, de l'irruption du sacré. Dans cette perspective, Durkheim propose le culte de l'humain qui « est devenu une chose sacrée, une sorte de dieu auquel on doit respect» (Piotte, 1999, p. 571). Dans ce culte de l'humain, Durkheim ne voit toutefois pas la vénération de «l'individualisme égoïste », mais la reconnaissance et la promotion de «l'humanité idéale».

Durkheim se réfère alors à la réalité substantive formant le principe intégrateur du sacré qu'il nomme le «principe totémique». C'est avec ce concept qu'il établit une analyse sociologique du sacré pouvant expliquer le comportement religieux de l'individu dans toute forme de société. Ainsi, on peut retenir que le religieux, chez Durkheim, ne s'exprimera pas uniquement dans les formes de religions traditionnelles, mais aussi dans d'autres lieux. De plus, cette théorie peut expliquer toutes les formes de relations que l'humain entretient avec les choses; en ce sens, le loisir peut être considéré comme une forme d'expression du religieux. Ce qui nous intéresse surtout dans l'explication du «principe totémique» pour saisir la radicalité du loisir, c'est l'élément intégrateur de l'expérience du sacré comme réalité substantive qui prend sa source dans la société elle-même. C'est l'ensemble des humains qui est la source du principe intégrateur de l'expérience du sacré. Durkheim précise davantage sa pensée où l'humain existe entre deux mondes, c'est-à-dire les mondes profane et sacré. Ce dernier monde le transporte hors de la vie ordinaire, dans la frénésie et l'extase. 
[...] On conçoit sans peine que, parvenu à cet état d'exaltation, l'homme ne se connaisse plus. Se sentant dominé, entraîné par une sorte de pouvoir extérieur qui le fait penser et agir autrement qu'en temps normal, il a naturellement l'impression de n'être plus lui-même. Il lui semble être devenu un être nouveau [...] Et comme, au même moment, tous ses compagnons se sentent transfigurés de la même manière et traduisent leur sentiment par leurs cris, leurs gestes, leur attitude, tout se passe comme s'il était réellement transporté dans un monde spécial, entièrement différent de celui où il vit d'ordinaire, dans un milieu tout peuplé de forces exceptionnellement intenses, qui l'envahissent et le métamorphosent. Comment des expériences comme celles-là, surtout quand elles se répètent chaque jour pendant des semaines, ne lui laisseraient-elles pas la conviction qu'il existe effectivement deux mondes hétérogènes et incomparables entre eux ? L'un est celui où il traîne languissamment sa vie quotidienne; au contraire, il ne peut pénétrer dans l'autre sans entrer aussitôt en rapport avec des puissances extraordinaires qui le galvanisent jusqu'à la frénésie. Le premier est le monde profane, le second, celui des choses sacrées (Durkheim, 1912, p. 312-313).

On relie souvent le jeu avec la survie et le combat: il «est une lutte pour quelque chose» (Huizinga, 1938, p. 35). Or, les communautés archaïques étaient régies par cette règle. À titre d'exemple, les activités de chasse s'accompagnaient de rites à caractère sacré tels que le dessin, le chant, la musique et la danse, de sorte que ces événements symbolisaient l'intégralité de la culture. Les images et les symboles de ces événements sont porteurs de la signification du lien social (Kelly, 1987). Les humains sont liés ensemble par le rite qui renforce l'engagement de chacun d'eux. Les activités de chasse, limités dans le temps et l'espace du jeu, représente le sens sacré des communautés archaïques. La culture est en un sens jouée (Huizinga, 1938). Cet état de chose peut se retrouver aujourd'hui dans les activités de loisir telles que le sport-spectacle et la fête.

Le sport-spectacle jouit d'une forte popularité : c'est un cadre spatiotemporel où un « sacré-intégrateur » réunit des foules considérables. La télévision joue même un rôle rituel en rassemblant des milliers de téléspectateurs. L'enjeu de la victoire du sport-spectacle rassemble les différentes classes sociales, en dépit des problèmes sociaux, et transporte dans l'enthousiasme, la fascination et, parfois même l'extase, les gens participant à l'événement compétitif. Le sport-spectacle met en jeu le sportif et le spectateur qui sont tous deux engagés dans la célébration de la puissance, de la beauté, de la vie, de la lutte et de la volonté qui crée une atmosphère religieuse (Bouet, 1968).

Coles utilise l'approche durkheimienne pour analyser le football européen, en tant que phénomène religieux, en affirmant que le rassemblement de plusieurs milliers de spectateurs, qui célèbrent leurs équipes, suppose les conditions d'une manifestation du sacré semblable aux rites d'expiation australiens. Ainsi, à travers la vivacité émotionnelle des humains en union intime dans les lieux sportifs, c'est la société elle-même qui s'affirme et se célèbre: «La foule a amplifié les sentiments et donne une puissance dans leur expression» (Coles, 1975, p. 68). 
Pour sa part, Brohm avance que les manifestations sportives telles que les Jeux olympiques ${ }^{9}$ peuvent s'assimiler à une forme de religion signifiante qui apporte un certain sentiment de fraternité et de paix aux humains qui y participent. Il va même jusqu'à affirmer que : «Le spectacle sportif supplante même en universalité toutes les religions dans la mesure où il s'adresse à tous les hommes, sans distinction de religion, à l'homme planétaire en général. Et les villes olympiques assument ainsi une vocation universelle plus signifiante que le Vatican ou la Mecque» (Brohm, 1976, p. 260).

Dans un article consacré à l'étude anthropologique du football européen, Augé (1982, p. 66) affirme que «quotidiennement la majorité des Occidentaux puisent la force de vivre dans [...] la sacralité laïque » qui se révèle dans le sportspectacle. Cette «sacralité laïque » se produit dans «l'instant» de la manifestation sportive, comme un rituel, où se réalise l'union intime des consciences individuelles. Elle permet de structurer un temps donnant ainsi un sens à la vie devant l'évanescence des dieux antiques.

Dans la culture de masse, le sport-spectacle est également une fête, qui peut être considérée à juste titre comme une «religion naturelle au $\mathrm{XX}^{\mathrm{e}}$ siècle». On y retrouve alors ce sacré que la société a égaré et qui se retrouve en particulier dans la nature (Isambert, 1982).

\section{Application de la théorie du sacré de Otto}

Otto a fait une description phénoménologique du sacré qu'il identifie à une sphère anthropologique spécifique se manifestant au-delà des catégories de l'éthique et du rationnel. Il en explique le caractère ambivalent. Pour lui, le sacré est la manifestation des forces psychiques subconscientes où se combinent le divin à l'humain, le rationnel et le non-rationnel. Otto (1917, p. 20) affirme que le sacré «apparaît comme un principe vivant dans toutes les religions. Il en constitue la partie la plus intime et, sans lui, elles ne seraient plus des formes de la religion ». Pour relever le caractère particulier de cet élément, Otto lui donne le nom de «numineux », un objet qui procure le «sentiment du mystère», et il l'étudie à travers de nombreuses manifestations des religions, des plus primitives jusqu' aux formes contemporaines du christianisme. Otto décrit le processus par lequel l'humain est saisi et découvre «pas à pas » l'objet numineux vibrant dans son âme. Le sacré, saisi comme élément numineux par l'humain et comme «principe vivant» des religions, est à la fois symbolique et mystique. Otto identifie quatre étapes de la saisie de l'objet numineux. La première implique le «sentiment de l'état de créature». Devant l'objet numineux, l'homme fait l'expérience d'un vif sentiment de dépendance, «[i]l s'abîme dans son propre néant et disparaît devant ce qui est au-dessus de toute créature»(Otto, 1917, p. 24). La deuxième étape est celle du mysterium tremendum, c'est-à-dire le mystère du «tout autre » qui angoisse l'humain au «tréfonds de son âme». Devant la majestas numineuse, l'expérience terrifiante 
prend la forme de l'effroi mystique. La troisième étape de la manifestation de l'objet numineux s'appelle le mysterium c'est-à-dire le sentiment de mystère. L'objet numineux se révèle comme le «tout autre » qui suscite le sentiment religieux véritable. C'est la transcendance qui se manifeste dans le «néant» des mystiques chrétiens ou le «vide» des bouddhistes. Finalement, la quatrième étape de la présence de l'objet numineux est le visage fascinant du mystère, c'est le mysterium fascinosum; c'est le mystère qui saisit l'humain dans une espèce d'enchantement, de fascination et d'émotion intime. De là découlent les sentiments d'émerveillement, de séduction, d'esthétisme, de béatitude, de compassion, de pitié et d'amour. Otto identifie cette expérience du numineux à ce que les religions institutionnelles nomment le salut ou l'expérience de la grâce.

\section{Enquête qualitative d'inspiration phénoménologique}

Afin d'appliquer la théorie du sacré de Otto à la pratique du loisir, nous avons mené une enquête qualitative d'inspiration phénoménologique. Or, la phénoménologie nous instruit que « la vraie philosophie est de rapprendre à voir le monde, et en ce sens une histoire racontée peut signifier le monde avec autant de "profondeur» qu'un traité de philosophie » (Merleau-Ponty, 1945, p. XVI). Maffesoli nous invite à prendre au sérieux l'approche phénoménologique, à «ne plus penser la vie sociale telle qu'elle devrait être, ou telle qu'on aimerait qu'elle soit, mais bien [...] telle qu'elle est. Il s'agit là d'une véritable révolution dans nos manières de penser » (Maffesoli, 1996, p. 151). Il ajoute que l'approche phénoménologique permet à la pensée d'ouvrir en profondeur jusqu'à la sensibilité de la vie quotidienne et de déboucher sur «la profondeur des manières d'être et des modes de vie postmodernes qui, de multiples manières, mettent en jeu des états émotionnels et des «appétits » passionnels reposant, largement, sur l'illumination par les sens » (Maffesoli, 1996, p. 266).

Chaque entrevue fut une expérience d'altérité pour le chercheur qui était persuadé que l'histoire de vie n'est pas simplement un récit d'expériences, mais également une activité communicationnelle composée d'errances créatrices et de hasards, soit les aléas de la recherche. Pour tout dire, «le récit [...] ne se contente pas de dire un mouvement. Il le fait. On le comprend donc si l'on entre dans la danse » (De Certeau, 1980, p. 154). Par cette activité communicationnelle à travers les histoires de vie, le chercheur a pu trouver ce qu'était le «monde social» des interviewés.

Pour mener cette enquête phénoménologique, nous avons réalisé des entrevues avec 21 personnes. Nous avons sélectionné des gens, hommes et femmes, jeunes gens et plus âgés qui pratiquaient une activité de loisir quelconque et qui avaient de la facilité à s'exprimer pour décrire leur histoire de vie le plus clairement et le plus complètement possible. Dans ces conditions, nous pouvions recueillir plus d'informations sur l'expérience du loisir qu'avaient les personnes interrogées. Voici l'ensemble des questions posées aux gens dans le cadre d'une entrevue : 
Pouvez-vous me décrire le plus fidèlement possible ce que vous « ressentez » lorsque vous êtes engagé à travers vos activités de loisir préférées ? Exprimez ce qui se «manifeste en vous-même»; énumérez ce que vous «pensez à l'intérieur » de vous-même. Indiquez les «sentiments » et les «impressions » que vous éprouvez au cours de cette expérience ainsi que vos intuitions, vos rêves, vos espoirs, vos valeurs. Par quoi êtes-vous « animé(e)»? Comment vous percevez-vous ? Comment vous « sentez-vous » avec les autres ou dans votre environnement ? Donnez, le plus souvent possible, des exemples précis de ce que vous décrivez. Également, décrivez une expérience où vous vous êtes senti(e) particulièrement dévalorisé(e), rabaissé(e) ou humilié(e) à travers une activité de loisir? (Deschamps, 1993, p. 57).

Le temps moyen nécessaire pour réaliser chaque entrevue était de trente à soixante minutes selon la personne. L'écoute active était de mise pour mettre en confiance notre interlocuteur et surtout pour recueillir «l'apparaître » de l'expérience du loisir le plus librement possible. Les entrevues furent enregistrées au magnétophone et retranscrites par traitement de texte à l'aide d'un dictaphone. À leur lecture, les données recueillies ont présenté à certains endroits une certaine redondance qui n'ajoutait plus, d'après nous, à la qualité d'informations désirées. Cet état de choses témoigne de la persistance d'une signification dans le récit de l'expérience du loisir. Nous avons alors décidé d'écarter les entrevues que nous avons jugées les moins significatives pour les besoins de la recherche (courte durée du témoignage ou propos manquant de consistance), si bien que le nombre fut fixé, comme corpus, à 17 entrevues.

La description phénoménologique implique «a priori » le respect d'une règle de conduite bien définie. Elle comporte nécessairement les étapes que le chercheur doit prendre en considération dans la mise en pratique de l'analyse des données qualitatives de l'enquête d'inspiration phénoménologique.

1. Observer le plus possible le verbatim de la description phénoménale, c'està-dire telle qu'elle est faite par la personne interviewée ;

2. Découper la description phénoménale en unités de valeur;

3. Émettre une description en termes d'analyse des unités de valeur.

À écouter les gens parler de leur loisir, on s'aperçoit qu'ils ont souvent recours à des catégories d'expression bien précises pour relater leur expérience. Les répondants décrivent leurs expériences de loisir le plus souvent en des termes à connotations spirituelles (Godbey et Gooddale, 1988, p. 238). À titre d'exemple, le loisir peut être vécu comme une activité de paix ou de joie. Ainsi, le sacré, bien qu'il persiste dans l'expérience des formes traditionnelles du religieux, peut aussi se manifester, et davantage, au cœur de l'expérience humaine du loisir dans des moments particulièrement riches et signifiants. L'humain vit alors une expérience paroxystique (Maslow, 1968). Parmi ces grandes expériences, mentionnons le sentiment de devenir soi-même, de participer aux énergies de la nature, de connaître le monde, de rechercher la beauté et d'aimer. Dans la logique de Otto, 
nous sommes alors portés à interpréter le loisir comme une expérience possible du sacré. Pour notre propos sur la manifestation du sacré dans le loisir, nous allons rapporter quelques discours du loisir qui expriment les émotions et les sentiments dans un cadre non religieux appliqués aux quatre étapes de la phénoménologie du sacré de Otto.

\section{Étape 1: Le sentiment de l'état de créature}

Dans les entrevues, certains ont dit se sentir petits devant l'immensité de la Création. Pour Jonathan, ce sentiment le ramène à beaucoup d'humilité face à l'immensité de l'univers. Ce qui n'est pas sans rappeler l'image philosophique ancienne du microcosme humain face au macrocosme du monde. Ce qui l'anime dans son expérience de loisir, c'est la recherche d'un état d'âme, de paix et de calme qui le rend content d'être petit, où il est heureux de faire partie de cet univers et de savourer l'harmonie qui en découle.

\section{Jonathan}

Comme on est petit face à la nature. Cela arrive régulièrement en plus des expériences de loisir. Les plus enrichissantes que j'ai vécues sont reliées à la survie en forêt où je partais avec un rien dans la nature, effectivement tout nu. [...] Pour moi, c'est important de retrouver cela pour me retrouver plus en contact avec la création. Je me sens petit. Le meilleur mot pour décrire cela me ramène à beaucoup d'humilité, très humble face à l'immensité de la chose. Et puis une humilité qu'on ne retrouve pas quand on est en contrôle parfait de la situation! Finalement ce contact-là est important parce que cela me ramène à cet état d'être petit. Il y a une forme de paix et de calme qui m'arrive. C'est cela qui m'amène de retrouver cet état d'âme. Ce qui m'anime, c'est de chercher l'état d'âme qui m'amène à être petit et être content d'être petit. Content de faire partie de cet univers. C'est cette recherche d'harmonie, cette recherche de faire partie de la nature d'être.

Il en est de même pour Karl qui dans l'immensité des paysages, éprouve un sentiment de petitesse. Dans l'activité de l'alpinisme, Karl est guidé par le principe du small is beautiful. Il aime la sensation que lui apporte la vie parce qu'elle est très importante et si fragile.

\section{Karl}

J'aime le risque. J'aime le défi. J'aime l'aventure. J'aime le « feeling» de ça. Mais ce que j'aime plus de tout ça, c'est le companionship, c'est l'esprit d'équipe, c'est le travail d'équipe, c'est le leadership. La paix que je retrouve. La grandeur des paysages. La petitesse que je retrouve en moi dans un monde impressionnant. J'aime le côté culturel des peuples que je rencontre. J'aime géographiquement voir des nouvelles choses. J'aime l'utilisation de mes sens. J'aime utiliser mes sens au maximum... des nouvelles odeurs, de sentir le froid, de sentir le soleil. J'aime la vie d'expédition.

André se sent également «petit» lorsque, comme professeur, il pratique la musique. Il est animé par la beauté de la musique qu'il qualifie d'innommable et d'impalpable. 


\begin{abstract}
André
J'admire les grands de la musique. Je veux toujours imiter les grands sans prendre conscience que j'ai atteint un certain niveau de performance et d'expérience. Pour moi, je suis encore un tout petit. Puis c'est intéressant parce que j'ai encore du travail à faire. Tu comprends mon point de vue ? En réalité, je suis animé par l'admiration, je ne dis pas l'adoration, mais la beauté, la bonté dans la musique. Qu'est-ce que la beauté dans la musique ?... c'est l'innommable, l'impalpable.
\end{abstract}

\title{
Étape 2: L'effroi mystique ou le mysterium tremendum
}

Dans son loisir, Karl fait mention de l'expérience de la peur, car lors d'une expédition d'alpinisme en haute altitude, il est confronté à ce phénomène. Bien que Karl sache qu'il peut mourir lors d'une expédition, il a appris à apprivoiser la peur de ce type de loisir.

\begin{abstract}
Karl
[...] Je me rappelle un jour. On était le groupe d'alpinisme ensemble qui partait pour l'Everest. On a fait un atelier complet sur nos craintes et nos peurs sur la mort. Ce qui nous faisait peur. C'est quoi nos plus grandes craintes qu'on avait afin de voir si on ne pouvait pas s'entraider là-dedans. On a discuté une journée complète sur la mort. Tout le monde dans le groupe ici avait vécu la peur de la mort. Comment on percevait cela ? On a appris à apprivoiser la mort. Le mot peur, c'est difficile pour moi. J'en discutais avec ma mère. Elle m'a déjà dit: «Tu n'as jamais eu peur dans ta vie. Tu n'as jamais eu peur, ni de la noirceur, ni de tomber, ni de personne, ni de la mort. » J'ai jamais eu peur. Par contre, j' ai des peurs. La peur, elle est présente. On y pense. Il n'y a pas un soir que tu es couché dans ta tente et que tu sais que le lendemain matin, tu pars pour 7000 ou 8000 mètres. Est-ce que mon corps va l'accepter? Est-ce que, physiologiquement, je vais manquer d'oxygène, que j'aille tomber en œdème ? Puis tu sais qu'en œdème, tu peux rapidement tomber dans le coma ? Comment ton système va réagir? Est-ce que je vais me faire prendre dans une avalanche? Je suis couché un soir dans ma tente puis il vente. Il commence à neiger. Est-ce qu'il va tomber trois pieds de neige et que peutêtre demain je sois pris dans la tempête ? Tu y penses, mais par contre cela fait partie du risque, de l'aventure...
\end{abstract}

\section{Étape 3: Le sentiment de mystère}

Si le loisir est un temps de repos, de détente et de relaxation, il est maintes fois associées à la méditation. Que ce soit dans des activités sportives ou de plein air, certaines personnes recherchent une paix intérieure; cela leur permet de se voir à l'intérieur d'elles-mêmes. C'est un temps privilégié pour réfléchir sur leur existence et son orientation. Le loisir les aide à se détendre et à se relâcher pour contrebalancer la vie trépidante de la société moderne. Ainsi, pour Karl, l'un des problèmes dans la société, c'est le manque de contacts de notre âme avec nos sources qui sont, d'après lui, en partie sensorielles. La tranquillité de la nature lui permet de méditer comme dans une église contrairement à la rapidité de la vie moderne qui ne donne pas le temps de penser. 
Karl

Dans mes loisirs, je recherche quoi ? Je recherche de me retrouver dans la nature, dans l'endroit où je suis paisible. Cela m'apporte, en même temps, à penser. C'est une méditation aussi dans un sens. C'est un genre de méditation de ce que les gens font à l'église. Ils ne prient peut-être pas durant une messe. Mais, durant un mardi après-midi, tu t'en vas à l'église, c'est tranquille, c'est paisible, c'est reposant, c'est calme. Tu as le temps de penser, de réfléchir à ce que tu fais en tant qu'être humain, etc. Moi, dans la nature, je retrouve les mêmes choses. Parce que c'est un environnement dans lequel je suis bien. C'est un environnement qui est paisible pour moi. Peu importe l'activité, je retrouve cette paix, cette tranquillité-là, ça me permet donc de penser à qui je suis, à ce que je fais, puis me reposer aussi. Faire le point et faire le plein d'énergie. Donc, mes activités de loisir préférées, les activités de nature se manifestent en moi surtout une paix, une relaxation, une détente, puis l'utilisation maximale de mes sens. En contact avec soi-même, tu es en contact avec le monde spirituel.

Pour Gabriel, l'expérience du loisir lui permet de se voir à l'intérieur de lui-même. C'est un moment privilégié de réflexion qui lui donne la possibilité de prendre les bonnes décisions sur l'orientation de sa vie. Le loisir l'aide à se détendre et à se relâcher. Son esprit devient plus clair et plus libre.

\section{Gabriel}

Tout le monde a besoin de se recueillir, de méditer, de s'asseoir à un moment donné pour penser à autre chose, à toutes ces valeurs naturelles. Les loisirs sont un autre aspect de notre personne qu'on peut montrer aux autres et puis quand on médite c'est la même chose. C'est voir à l'intérieur de nous autres ce que l'on ressent. La méditation est un troisième volet de la personne. C'est un besoin d'une personne de s'asseoir, puis d'être seule, à un moment donné, et de réfléchir à sa vie et son orientation. [...] Le loisir va t'aider dans le sens qu'il va permettre de te détendre, de te relâcher, d'oublier les choses après une activité de loisir. Ton esprit devient plus clair, plus libre, tu peux alors mieux méditer. Le loisir bien souvent permet de faire un petit résumé de ce qui se passe dans notre vie. Cela nous donne le temps d'espérer pas seulement au point de vue matériel mais aussi au point de vue spirituel. Tu te sens plus détendu, mais, à ce moment-là, tu te permets de réfléchir aux affaires plus terre-à-terre. Qu'est-ce qu' on est comme personne ? C'est fait pour travailler, c'est fait pour s'amuser, mais aussi, c'est fait pour méditer.

\section{Étape 4: L'expérience fascinante ou le mysterium fascinosum}

Pour Anne, le ski alpin comme expérience de loisir lui procure à la fois de la détente, de la relaxation ainsi que de la joie et de la liberté où elle se laisse aller légèrement à ses fantaisies.

\section{Anne}

Le ski alpin est mon loisir. Ce que je ressens quand je fais mes loisirs, c'est de la détente, de la relaxation, de la liberté, c'est de la joie... je suis heureuse. La vie est belle et bien agréable. [...] En ski alpin, c'est la liberté totale. Tu t'en vas où tu veux, c'est le vent des fois, c'est le froid quand il fait beau. $\mathrm{Tu}$ te laisses aller. C'est sûr que tu contrôles tes mouvements. Mais moi, 
souvent, j'ai l'impression de valser dans certaines pentes. Alors, j'entends comme une musique dans ma tête. J'ai l'impression de valser. Puis, j'entends ma musique dans ma tête avec mes mouvements. C'est la liberté totale. C'est laisser tout aller. C'est entre mes deux oreilles. [...] La neige est à un certain degré et la pente est à une certaine qualité qui me plaît. Je l'appelle ma valseuse puis quand je descends, j'entends la valse des patineurs, puis je m'en vais.

Certaines personnes éprouvent un sentiment d'admiration dans leur pratique de loisir; c'est le cas d'André dans son expérience de la musique. En écoutant de la musique, il est vraiment ému. Tout est dans l'admiration de la créature. Il s'interroge, car l'admiration est tellement intense et universelle qu'il « s'incline » devant la grandeur du Créateur et de sa créature qui a composé une si belle musique. Voici comment il décrit son expérience de loisir dans la musique qui est de nature esthétique.

\section{André}

J'écoute une grande musique, puis là vraiment, j'essaie de me rentrer dedans, l'admiration est tellement grande du compositeur. Excuse l'expression anglaise: «Je capote, je tripe, je pars!» Je pars en voyage dans un sens que c'est un voyage admiratif. Et moi, je ne peux pas m'empêcher de penser au Créateur de ce génie-là. C'est une déformation professionnelle, c'est un peu normal, mais j'en ai rencontré d'autres et c'était pareil. Vraiment, on s'interroge, puis l'admiration va tellement haut, tellement grande [sic] C'est tellement étendu, tellement universel que là vraiment, comme dirait quelqu'un : «on s'incline». [...] En écoutant de la musique, je suis vraiment ému, oui, mais, ce qui m'étonne de plus en plus, ce n'est pas une émotion sentimentale. Il y en a qui pleurent, moi je ne pleure pas. C'est tout dans l'admiration. C'est une admiration devant la créature. Le Créateur et la créature. Parce que, moi, on admire ce que je fais en musique, ça me fait ben drôle. Parce que j'écoute Beethoven, puis moi, je me dis là, que simplement pour me mettre dans la tête. J'ai une partition d'une symphonie, la symphonie $\mathrm{n}^{\circ} 9$, qui est un sommet, jusqu'à un certain point. Tu as quatre solistes làdedans, tu as une chorale de quatre ou cinq voix. Et ensuite, tu as tous les instruments l'un après l'autre. Comment un bonhomme... ? Non, vraiment ça me dépasse ! C'est un peu comme des couchers de soleil le soir, pis là tu es dans la stratosphère, tu essaies d'imaginer les étoiles, tu te perds net... Tu es parti ! C'est à peu près la même chose, mais il y a un côté admiratif, parce que c'est dans des personnes que tu côtoies par tes oreilles.

Pour Claude, le golf comme expérience de loisir propose un milieu agréable dans des sites enchanteurs. Dans ce lieu, il y retrouve toute la beauté de la nature qui représente, pour lui, un bonheur.

\section{Claude}

Au golf, tu es dans des sites enchanteurs. C'est relaxant, c'est reposant, c'est vert, c'est beau, c'est joli, en général. On est sur un terrain de golf. Il fait beau soleil. C'est bien aménagé, c'est entretenu, c'est vert. Il y a des lignes d'arbres, des fleurs, de l'eau. Le gazon est à la bonne hauteur. Puis on commence à jouer au golf et ça fait qu'après un ou deux coups on se dit: « On est-tu bien ? 
C'est beau! Peut-tu demander mieux ? La nature est belle ! [...] Le gazon et les allées verts, c'est magnifique. Je suis heureux. Je rencontre des amis dans des conditions agréables, dans une activité que j'aime. C'est un bonheur...

À côté des praticiens du loisir, il y a des spécialistes qui ont montré que toute une série d'objets peuvent être porteurs du numineux. À titre d'exemple, on peut observer à travers les «vedettes du sport ou de faits divers, «stars » des milieux du spectacle font l'objet d'une fabrication d'images numineuses, source de véritables cultes ou rassemblements liturgiques. Leur image ou leurs paroles jouent le rôle de reliques ou d'oracles, leur vie est source de fabulation mythique rejouant d'antiques scénarios sexuels ou conflictuels, leur succès, leur prestige ou leur richesse sont vénérés comme des attributs de puissance exceptionnelle» (Wunenburger, 1981, p. 122). Certes, il peut être tentant d'identifier ces phénomènes à l'idolâtrie et au fétichisme. Toutefois, au-delà de tout discours moralisateur, il faut être plus attentif à cette manifestation du sacré, de cette charge numineuse qui s'inscrit dans la réalité du loisir de consommation et attire irrésistiblement le monde à elle.

\section{Pour une mythologie du loisir}

Pronovost soutient que le mythe du loisir « se rattache à un nouveau monde, à venir ou en voie de réalisation. Il a une portée eschatologique. Le loisir implique, recèle l'affirmation d'une nouvelle forme de société, dont le contenu est identifiable aux grands thèmes déjà présentés : identité retrouvée, santé, naturalité » (Pronovost, 1997, p. 43). Ainsi, à travers la symbolique que déploie le sport-spectacle, le principe intégrateur du sacré est porteur d'identité; les gens peuvent s'identifier à tel athlète ou à telle équipe. Les superathlètes deviennent, bien malgré eux, les nouvelles idoles ${ }^{10}$ jouant ainsi la vie des héros mythiques.

Cette dernière affirmation va dans le sens de Dufour qui développe une mythologie du loisir. Pour lui (1977, p. 22), « le loisir, qui semble futile à première vue, est un acte qui se vit «religieusement», puisque c'est le temps prodigieux, fabuleux, sacré». Ainsi, il montre qu'en dépit de l'effritement des religions institutionnelles le sens du sacré chez l'humain de l'Occident continue à se manifester sous une forme mythique fondamentale. Dufour reprend à son compte la description du mythe romain de Bacchus (Dionysos pour les Grecs) pour démontrer la prééminence du sacré dans le loisir aujourd'hui.

À l'époque classique, Bacchus prit l'allure de la vie joyeuse, des jeux, des fêtes, des danses et des transports frénétiques et désordonnés. Le week-end moderne est aussi une fête qui donne lieu à toutes sortes de bacchanales qui tournent même à l'orgie sous l'effet de l'alcoolisme ou des diverses drogues. Cette quête d'ivresse s'exprime de bien des manières : vitesse de la moto ou de l'auto, manèges des foires, déviations sexuelles, pornographies, obscénité et prostitution. Il y a une sorte de démence des fêtards : la fureur de vivre. [...] La taverne ou le « pub», surtout dans les milieux populaires, est un endroit 
très fréquenté en week-end. Si c'est une occasion de dépense, c'est avant tout un lieu de rencontre entre les hommes. Une dynamique tisse un réseau d'amis où, autour d'un verre, on discute, se valorise, retrouve une certaine assurance. La taverne ou le café remédie à la solitude et au manque de sécurité personnelle. Une autre fonction de ces points de chaude camaraderie, c'est qu'on tente de «régler» provisoirement ses problèmes en les oubliant. [...] «Vivre selon » un mythe, en l'occurrence celui de Bacchus, c'est cesser d'exister seulement dans la vie quotidienne. Les rites de la fête amorcent une sorte d'intériorisation émotionnelle qui engendre le noyau mytho-poéthique [sic] indispensable à toute existence humaine. (Dufour, 1977, p. 221, 237-238)

Personnage étrangement ambigu et ambivalent, Dionysos est l'idéal présenté par Nietzsche qui cherche à promouvoir l'affirmation de la vie et non sa négation. Il cherche à se libérer d'une morale rationnelle et extérieure, du sentiment de culpabilité et du ressentiment imposé par un monde de production excessive. Dans le monde actuel, en Occident, Dionysos se révèle en quelque sorte un nouvel être-au-monde, car le jeu, le loisir et la fête procurent un style de vie caractéristique de la postmodernité.

Pour terminer cette métaphore, on doit d'abord reconnaître qu'il est toujours difficile pour les non-spécialistes d'identifier le sacré avec le loisir ou le jeu. Il en est de même pour les relations entre le sacré et Dieu ainsi que pour les relations entre Dieu et le loisir ou le jeu. Ainsi, Platon n'hésitait pas à réunir «l'action sacrée » et le jeu qu'il opposait toutefois au sérieux. Dans sa logique, ce lien résultait de la volonté du Créateur, Être sérieux par excellence qui assigne à l'humain un rôle ludique. Ainsi, pour gagner la faveur de Dieu, l'humain doit se cantonner dans le jeu et s'abstenir de poser tout acte sérieux, comme la guerre par exemple ${ }^{11}$. Platon crée une frontière en mettant Dieu du côté du sérieux et en réduisant l'humain à être l'objet de Dieu : «un jouet». En ce sens, la grâce que son Dieu offre à l'humain est très limitée ; il donne plutôt l'image d'un Deus faber.

Cette théorie platonicienne a un mérite certain, car elle permet de reconnaître des liens entre l'action sacrée et le jeu, mais ces liens ne sont pas toujours évidents aujourd'hui, le sacré étant souvent considéré comme quelque chose de sérieux. Il implique des lois, des rites, des interdits auxquels l'humain doit se soumettre. Dans cette perspective, il y a une rupture entre la gravité du sacré et la légèreté du jeu. Le sérieux du sacré devient donc l'antithèse de la métaphore du « religieux ». Cette antithèse répond à un modèle de production et de gestion : il faut faire des rites pour faire des rites et obéir à la structure des normes et règlements d'une institution religieuse. Les religions traditionnelles peuvent entretenir le sentiment de culpabilité dans un discours d'une morale du malheur, de la punition et de l'idéal ascétique et limiter le religieux à l'obligation. En fait, c'est l'étouffement des désirs de l'humain et sa réalisation créatrice. Lorsque le sérieux du sacré s'enferme dans une dynamique fonctionnelle et restrictive, sa circulation est peut-être menacée ; l'humain est alors emprisonné dans une «cage de fer» et doit se plier aux règles institutionnelles (Drewermann, 1993). 
On peut dès lors se demander si les religions peuvent présenter une ouverture au loisir comme mode d'existence. Les théories présentées dans cette métaphore $\mathrm{du}$ «religieux » demeurent, somme toute, très générales. Or, le sacré ne peut pas être traité dans une seule approche générale. En fait, il peut se vivre d'une manière diffuse, mais, dans la plupart des cas, il se vit surtout dans une religion particulière. De plus, il n'est pas possible pour nous d'explorer la richesse ludique de toutes les religions traditionnelles de sorte que nous allons nous restreindre à notre propre tradition qui est celle du christianisme. À partir de là, on peut se demander s'il est possible, dans le risque d'une foi qui cherche à comprendre, de trouver dans le christianisme un Dieu ludique voulant notre bien en jouant avec nous et de poser les mêmes questions de Dumont (1995, p. 212) : «Ne faut-il pas, [...] qu'une société se considère comme perpétuellement inachevée, qu'elle avoue ses manques et sa finitude ? N'est-il pas nécessaire, pour tout dire, qu'elle puisse se juger face à une transcendance? » Dans cette perspective, la métaphore de la «transcendance» s'intéressera de développer, dans une vision du christianisme, la possibilité de la révélation d'un Dieu ludique, le Deus ludens.

\section{La métaphore de la «transcendance»}

Cette section se veut un prolongement du modèle théorique de Kelly. Dans la métaphore précédente, l'antithèse du sérieux du sacré s'oppose au jeu, à l'essence même du loisir. Pour Kelly, «la vraie réalisation de la société industrielle devrait être utilisée comme base économique pour une société cherchant la grâce plus que la possession et le jeu plus que la production » (Kelly, 1987, p. 223). Il en va de même pour l'humain, qui confronté au sérieux du sacré, a besoin de la grâce ${ }^{12} \mathrm{du}$ Dieu ludique ${ }^{13}$ pour s'orienter dans le processus créateur du devenir. Ainsi, la grâce est la thèse de la métaphore de la «transcendance » qui veut instaurer une relation ludique de la personne de Dieu avec les humains dans leur vie concrète. La grâce de Dieu est un don gratuit qui, accueilli dans la foi, régénère et reconstruit l'humain; le don de la grâce permet de nous introduire en toute liberté dans l'amitié et l'intimité de Dieu. Dans la métaphore de la «transcendance», il s'agira de découvrir un Dieu de grâce qui se révèle par le jeu dans l'acte de la création. Cette métaphore traitera donc de l'importance que prend le loisir et le jeu dans une approche anthropologique judéo-chrétienne. En voici une vue d'ensemble.

Quelques clés conceptuelles de théologie chrétienne permettent de prendre conscience davantage de la réalité divine dans la pratique du loisir. Ainsi, la révélation de Dieu dans l'histoire de la création et du salut, la révélation transcendantale selon Rahner et une théologie du jeu de la création et du salut d'après Moltmann s'avèrent importantes dans le développement d'une relation ludique entre Dieu et le pouvoir créateur de l'humain. En ce sens, le jeu est compris comme symbole de la création, et, par le fait même, de la réalité du monde, de la réalité de l'humain, du Créateur lui-même et de leur relation mutuelle. Il est l'environnement où l'humain peut participer à l'agir de la création et du salut par anticipation du Royaume à advenir. La relation au Dieu ludique demeure cependant très fragile en raison de l'antithèse de la dé-création. 


\section{La révélation de Dieu dans l'histoire de la création et du salut}

Pour mieux saisir la radicalité du loisir, la notion de la révélation de Dieu dans l'histoire de la création et du salut nous semble être une clé d'interprétation théologique essentielle. Notre intention ici n'est pas de formuler un discours dogmatique sur la révélation, mais d'essayer surtout de dégager quelques éléments de compréhension élaborés par des théologiens qui établissent des liens avec le loisir et le jeu. Certes, il n'est pas si facile de considérer la révélation de Dieu dans l'histoire de la création et du salut à travers les événements humains de la vie quotidienne. Un découpage s'impose. Ainsi, le premier lieu de la révélation concerne tout d'abord la vie même des humains : «Si Dieu parle, alors c'est $\grave{a}$ travers l'homme et dans l'homme avec tout son monde et toute son histoire; c'est dans ce tout que nous ferons entendre la voix de la révélation de Dieu » (Schillebeeckx, 1968, p. 106).

La révélation est comprise comme une nouvelle prise de conscience et un engagement de l'humain par rapport à l'action transcendante de Dieu qui se fait connaître dans l'histoire: «il se révèle dans l'histoire humaine, que déterminent son alliance et sa promesse ( Moltmann, 1988, p. 27). Autrement dit, «Dieu se révèle lui-même en révélant l'homme à lui-même ». L'expérience de la révélation est en même temps une expérience de création et de salut, un processus de vie plutôt qu'un message tout fait. La révélation permet l'espace-temps d'une rencontre avec le Dieu transcendant.

Pour sa part, Rahner a bien clarifié la façon dont nous faisons l'expérience simultanément de nous-mêmes et de l'ouverture à la transcendance qui est offerte à tous les humains. En effet, toute expérience humaine a une ultime dimension religieuse, qui est la forme essentielle de l'autocommunication révélatrice et salvifique de Dieu que Rahner nomme la révélation transcendantale. Dans la révélation de Dieu dans l'histoire de la création et du salut, on peut affirmer que Dieu est transcendant au sein de son immanence. Ainsi, «[...] il est impossible de penser la transcendance divine par rapport au monde sans cette immanence divine dans le monde, comme à l'inverse il n'y a pas d'immanence évolutive de Dieu dans le monde sans sa transcendance par rapport au monde. Les deux sont en relation réciproque ( (Moltmann, 1988, p. 266).

Dans sa théorie de la révélation transcendantale, Rahner développe la notion innovatrice de l'autocommunication de Dieu comme créateur au monde qui réalise l'autotranscendance du monde en lui et vers lui. L'autocommunication de Dieu devient alors un mystère intime qui vient habiter la matière. Pour Rahner, «Dieu ne crée pas seulement ce qui est distinct de lui, mais il se donne lui-même à ce qui ainsi est distinct de lui. Dieu, le mystère infini et indicible, le monde le reçoit à ce point qu'il devient lui-même sa vie la plus intime » (cité dans Moltmann, 1993, p. 406). Pour sa part, Ebeling approfondit le concept de révélation en lui attribuant 
une nature esthétique qui contribue à intégrer l'expérience de la transcendance à travers l'expérience du loisir sans référence au domaine religieux traditionnel (Waldenfels, 1990, p. 253-254).

De tout ce qui précède, la question qui se pose est la suivante : comment faut-il comprendre le fait que la révélation transcendantale puisse être universellement accessible à tous les humains ? Geffré donne quelques éléments de réponse à cette question. Il parle de l'expérience de différents «états de conscience » qui sont des acquis indéniables du devenir historique de l'humain. Ces «états de conscience» sont « le sens de l'autonomie de la conscience, l'aspiration au bonheur, la dignité de la personne et le prix de la vie humaine, la conception démocratique de la vie en société, le droit à la liberté religieuse et l'acceptation du pluralisme» (Geffré, 1990, p. 13). La notion des «états de conscience» rejoint du même coup la pensée de Peelman (1996, p. 40) qui affirme que «la révolution culturelle que nous vivons depuis la dernière guerre mondiale est absolument globale. Elle a produit un nouvel environnement culturel et une nouvelle conscientisation globale». Ces «états de conscience » et cette «conscientisation globale » qui s'expriment particulièrement dans «l'aspiration au bonheur» deviennent, pour notre part, le temps et le lieu privilégiés de la révélation du Dieu transcendant dans l'expérience humaine du loisir. Autrement dit, l'autocommunication de Dieu dans l'expérience historique du loisir peut devenir le temps et le lieu de la révélation que Dieu fait de Lui-même dans la grâce christique. Cette révélation transcendante et immanente de Dieu dans l'histoire de la création et du salut peut prendre la forme d'une dynamique ludique avec l'humain.

\section{Théologie ludique de la création et du salut}

On a souvent une vision très réductrice de la création en la limitant à l'origine de la nature. Or, la création pour Dieu n'est pas seulement une œuvre qui a été faite en sept jours. Certes, les récits de la création exprimés dans la Genèse révèlent le Dieu Créateur, Un et Universel. Mais il faut aussi parler, dans le processus ininterrompu de l'histoire du salut, du Dieu de l'Exode et de l'Alliance qui se manifeste dans sa création : «Dieu ne fait pas que créer : il se communique également d'une certaine manière à ses créatures, car par l'Esprit et la Parole le Créateur entre dans sa création et la pousse en avant» (Moltmann, 1993, p. 410). Ainsi, la création suit le cours de l'histoire et l'humain est appelé à y participer. Elle est aussi la grâce d'être saisi par Dieu. Comme fondement de l'être, Dieu est également le fondement de la puissance de l'amour créateur de l'humain, ce qui rejoint Kelly pour qui le jeu de la création permet à l'Esprit du Créateur d'accéder à une plus grande connaissance de sa nature (Kelly, 1987, p. 214).

On peut dire qu'il en est de même pour l'humain qui, à l'image de Dieu, est un projet de sens, un projet de création à travers l'exercice de sa liberté dans ses relations avec Dieu et avec les autres. Déjà Berdiaev disait que Dieu dote l'humain 
d'un pouvoir créateur dans le monde: «Dieu attend de l'homme la découverte anthropologique de la création » (cité dans Davy, 1964, p. 111). Dieu cherche la liberté de l'humain, c'est-à-dire la réalisation libre de son pouvoir créateur. Trop longtemps, les discours théologiques dominants ont occulté le pouvoir créateur de l'humain en mettant l'accent sur ses faiblesses. S'inscrivant en faux contre cette position, Camus explique qu'il faut essayer de conquérir l'être, de le créer à partir du peu d'être que nous découvrons en nous et non pas de le nier au départ. L'humain créé à l'image de Dieu possède en lui-même une capacité créatrice participant de l'acte créateur. Dès lors, l'acte de création peut prendre un sens à la fois divin et humain dans ce que Berdiaev définit comme des "percées créatrices », c'est-à-dire des perspectives nouvelles de l'univers ou de la découverte des mondes inconnus (Davy, 1964).

Dieu est le fondement créateur de l'être; il est au cœur de toute liberté humaine. C'est dans la dynamique de l'alliance avec Dieu que nous héritons d'un pouvoir créateur. Dieu crée l'humain créateur, mais celui-ci a la lourde responsabilité de collaborer à sa propre création existentielle. Dieu agit dans l'acte de foi où l'humain se réalise tout en étant distinct de Dieu, et, en même temps, participant au processus de la création et du salut. Ainsi, l'humain créateur doit s'exercer à transformer le néant en plénitude. L'humain doit apprendre à développer son pouvoir créateur, car il est appelé lui-même à la transcendance.

$\mathrm{Si}$ « la création de l'homme poursuit l'œuvre de la création de Dieu» (Davy, 1964, p. 111), peut-on parler de l'œuvre de la création comme d'un jeu ? Si, d'après Faure, «Dieu est un enfant qui s'amuse », on pourrait affirmer, étant ses enfants, que nous jouons avec Lui le jeu dans la liberté de la création et du salut. Plus profondément, on peut dire que l'aspect esthétique du jeu fait participer l'humain à l'histoire et au salut. Moltmann (1977) a développé une compréhension théologique et anthropologique du jeu. Pour lui, le jeu est un symbole cosmique du monde puisque le jeu peut être une illustration qui explique le mieux son origine; le jeu fait advenir l'humain au monde.

Le «jeu comme symbole du monde» permet de comprendre l'existence humaine dans le temps du monde. C'est bien souvent en jouant que l'on se libère du poids des contraintes de l'existence et que l'on s'amuse à voir les choses non pas comme elles sont, mais comme on pense qu'elles devraient être. Dans le fragment 52, Héraclite affirme que «le temps du monde est un enfant qui joue et qui place les pions ça et là; c'est un royaume de l'enfant». Dans l'acte de jouer, il n'y est plus question de maître et de valet, on se retrouve comme un enfant dans toute la profondeur de la confiance originelle dans une création qui peut s'interpréter dans le jeu de la vie. Ainsi, la foi se découvre dans la confiance originelle puisque le monde appartient au «royaume de l'enfant». La «naïveté sérieuse » de l'enfant évoque la dimension éphémère et relative des choses de la «totalité du monde ». Pour paraphraser Volant (1976, p. 82-83), l'humain est un processus 
en devenir ayant la faculté d'établir une distance critique par rapport à lui-même. Il peut s'imaginer et se vouloir autrement de ce qu'il est aujourd'hui. Dans cette perspective, il joue avec son existence. Le jeu prend donc le pas sur l'absurde et permet d'aller du non-être à l'être. Autrement dit, par le jeu, l'humain a la capacité de faire advenir son existence dans son intégralité en déployant, en toute liberté, ses possibilités réelles. Cet «acte existentiel » crée la dynamique interne de toute «praxis ludique». Le jeu devient alors un acte de la volonté dans la liberté. Puisque l'essentiel pour l'humain «c'est d'être jouant», on peut parler de lui comme d'un homo ludens (Huizinga, 1938).

Pour Moltmann, le jeu de l'existence est la joie éternelle de Dieu qui fait jouer et danser toute la création. Pourquoi Dieu a-t-il créé le monde ? Il ne l'a pas fait exclusivement pour le travail ni pour gagner quelque chose en créant les humains. La création est une action libre, volontaire et gratuite du bon plaisir de Dieu; elle est une action de la volonté amoureuse de Dieu, non un «accident de parcours » ni un « hasard ». C'est une manière de manifester la divinité et la grâce de Dieu. Moltmann emploie la notion de creatio ex nihilo ${ }^{14}$ de la tradition judéochrétienne pour illustrer la gratuité du jeu créateur de Dieu. C'est «le Dieu qui fait vivre les morts et appelle à l'existence ce qui n'existe pas» $(\mathrm{Rm} 4,17)$. Par ailleurs, Moltmann est conscient que le jeu qui s'inscrit dans la contingence des événements mondains peut être fréquemment expérimenté par l'humain comme ayant une dynamique cruelle s'il ne fait pas confiance à une providence.

Dans la métaphore du «religieux», on a vu que le Dieu de Platon est un «dieu sérieux» et que l'humain y est considéré comme le «jouet de Dieu». Celui-ci n'est pas perçu comme un Dieu de grâce qui créé un humain capable de créativité; au contraire, l'humain est créé pour être un «jouet de Dieu». Dans ce cas, serions-nous alors de simples marionnettes de Dieu? Le cours du monde est-il une grande pièce de théâtre, une grande fresque parodique, où "l'hommejoueur » et «jouet de Dieu» est l'acteur jouant son propre personnage, un petit rôle en trois actes où se déroule la toile de fond de son existence : naissance, vie et mort ? La théorie platonicienne prive Dieu de sa grâce, et, dans cette perspective, nous ne sommes sans doute que des marionnettes, mais comme chrétiens, nous trouvons qu'il y a un espace de liberté qui laisse à l'humain la possibilité d'être, de créer et de jouer. Ainsi, le jeu peut prendre la forme d'une libre relation avec JésusChrist : «C'est pour que nous soyons vraiment libres que Christ nous a libérés » $(\mathrm{Ga} 5,1)$. Le jeu permet à l'humain de devenir libre dans le Christ et non de s'aliéner en se perdant dans le jeu. De sorte que le lien de Platon est fait pour être défait avec la libération de la grâce christique.

L'action ludique de Dieu appelle l'humain à sa pleine réalisation. À travers le jeu de la création, il vit un processus de libération par lequel il puise suffisamment de joie pour accéder pleinement à la vie. L'humain apprécie déjà, par anticipation, la joie d'être libre dans le Christ. Dans cette perspective, l'humain devient 
un homo ludens. Guitton qui invoque la sagesse de monsieur Pourget affirme que: «La liberté est le pouvoir de devenir ce que nous devons être » (cité dans Foulquié, 1962, p. 405). C'est pourquoi le jeu de l'humain comme partenaire du jeu créateur de Dieu n'a d'autre orientation possible que dans la pleine réalisation de sa liberté. L'humain est appelé à sa réalisation: «Être appelé à la vie de la raison et de la liberté, c'est participer à la libre nécessité de Dieu qui ne peut manquer de se vouloir. Nous aussi, nous ne pouvons manquer de nous vouloir» (cité dans Foulquié, 1962, p. 405). Le jeu est la quête de la liberté, une revanche sur l'inéluctable fatalité de l'existence.

On en revient à la métaphore «humaniste» de Kelly qui pose cette question: «Comment l'humain devient-il humain?» La réponse de Moltmann est la suivante : l'humain ne peut pas devenir humain uniquement par le travail ; l'humain peut se découvrir dans l'agir libre du jeu gratuit et créateur de Dieu selon son bon plaisir. En définitive, l'humain comme enfant de Dieu est créé pour jouer. Il recherche le plaisir et la joie qu' apporte en lui-même l'acte de jouer qui devient alors une fin en soi.

À travers l'expérience du jeu, surgit alors la question d'une spiritualité ${ }^{15} \mathrm{du}$ loisir qui permet d'être saisi par la grâce donnant un sens à la vie. Cette grâce provenant de la transcendance inconditionnelle se traduit par une émotion de profondeur qui procure la joie de vivre. Ainsi, dans cette spiritualité du loisir, il y a cette possibilité que l'humain soit saisi dans ce que Dubos (1974, p. 200-201) appelle la joie de vivre, une joie à la fois organique et créatrice. Cette façon de percevoir l'Inconditionnel est confirmée par Boff qui voit dans les célébrations, en particulier celle de Noël, une fête pour les cœurs d'enfant: «La foi devient sentiment. Elle atteint par là ce qu'il y a de plus profond et de plus intime dans la personnalité humaine : elle fait vibrer, elle apporte la joie, elle fait goûter la vie en lui donnant un sens » (Boff, 1985, p. 162). Moltmann (1988, p. 100) ajoute que « la perception du monde comme création suscite la joie de vivre. La présentation du monde à Dieu dans l'action de grâce suscite la liberté de vivre ». Ce qui rappelle la pensée de Bergson $(1919$, p. 23) : «[...] la joie annonce toujours que la vie a réussi, qu'elle a gagné du terrain, qu'elle a remporté une victoire: toute grande joie a un accent triomphal. Or, si nous tenons compte de cette indication et si nous suivons cette nouvelle ligne de faits, nous trouvons que partout où il y a joie, il $\mathrm{y}$ a création : plus riche est la création, plus profonde est la joie».

L'antithèse de la métaphore de la «transcendance» se manifeste dans la dé-création qui comporte deux aspects. Tout d'abord, il y a la difficulté de vivre le paradoxe où l'humain est soumis au danger de l'absurde (révolte et/ou pessimisme), et, en même temps, d'y réagir par une ouverture à Dieu. L'humain a de la difficulté à assumer ce paradoxe. Tel un funambule, il est sur une corde raide. Il faut être capable de pratiquer le loisir et le jeu malgré les difficultés de la vie, sans quoi, il y a danger de régression et d'enfermement dans l'image du «Dieufaber». Le «Dieu-faber» est le grand horloger qui oblige l'humain à fonctionner 
selon une programmation prédéterminée; son plan divin n'inclut pas la liberté. Dans ce contexte, l'humain ne peut plus jouer librement avec le Créateur. Le deuxième aspect de la dé-création peut se traduire en des termes théologiques sous le thème du péché. Dans une approche rahnérienne, «le péché est l'état où nous ne savons plus ce que nous sommes parce que nous sommes coupés de la communication que Dieu fait de lui-même» (Williams, 1998, p. 874). En fait, le péché signifie une rupture avec Dieu. Sans le don gratuit de la grâce régénératrice, l'humain est confronté, souvent bien malgré lui, au péché qui produit la mort dans son âme. Dans ces conditions, la relation ludique avec Dieu est brisée et, sans son intervention secourable, elle n'est plus possible.

La spiritualité est l'une des composantes importantes du développement de la personne et, en ce sens, il peut être un facteur déterminant de bien-être et de santé mentale dans son processus de vieillissement. Déjà des recherches sérieuses établissent plusieurs liens entre l'expérience du loisir et le bien-être spirituel (McDowell et Schreyer, 1991; Heintzman, 2000). Cette présente étude expose donc les prémisses d'un cadre théorique pour un discours sur la spiritualité du loisir et propose une approche nouvelle et particulière pour aborder le loisir ; elle pourrait être de plus en plus d'intérêt pour l'avenir notamment en ce qui concerne l'éducation au loisir et le loisir thérapeutique.

\section{NOTES}

1. Cette étude est un texte remanié de deux chapitres d'une thèse de doctorat en théologie pratique à l'Université de Montréal qui s'intitule: Pour une spiritualité du loisir créateur: Essai de théologie pratique. Dans le contexte d'un monde pluraliste, la postmodernité favorise une nouvelle approche théologique qui se nomme la théologie pratique. Pour mieux comprendre l'agir chrétien en société dans une dialectique entre théorie et praxis, la théologie pratique doit s'inscrire dans une approche interdisciplinaire. La théologie pratique s'inspire de la méthode praxéologique qui part de la pratique elle-même qu'elle questionne, interprète tout en suggérant des moyens d'intervention. Elle comprend différentes coordonnées : l'observation, l'interprétation, l'opérationnalisation de l'action et la prospective.

2. John R. Kelly (1987). Freedom to be : A new sociology of leisure, New York, Macmillan. Cet ouvrage est le fruit d'une riche expérience universitaire dans les champs d'études du loisir. Ancien professeur au Département des études du loisir à l'Université d'Illinois (Urbana-Champaign), Kelly a aussi été professeur à l'Institut de recherche du développement humain ainsi que directeur de l'Office de gérontologie. Il a publié plus de huit ouvrages qui s'intéressent aux questions de la signification du loisir à travers le parcours de vie, les contextes de changement de rôle et l'interaction sociale. Chef de file de la World Leisure and Recreation Association (WLRA), il est conférencier principal dans plusieurs congrès de recherche. Il est aussi un scientifique œuvrant auprès des groupes sociaux et un consultant pour le gouvernement américain. Son livre Freedom to be lui donne surtout la réputation d'un sociologue existentialiste. 
3. On commence, ici au Québec, à se référer au modèle théorique du loisir de Kelly. Voir Jean-Louis Paré (1997). L’intégration du migrant par les loisirs, Pourquoi partir? La migration des jeunes d'hier et d'aujourd'hui, Québec : Presses de l'Université Laval, p. 193-194.

4. Pour Kelly, le jeu et le loisir sont des activités similaires lorsqu'on les définit tout d'abord comme une action choisie afin de vivre une expérience positive.

5. Pour ces deux métaphores, nous adopterons la démarche intellectuelle de Kelly, soit celle qui consiste à utiliser plusieurs auteurs et termes pour expliquer sa théorie. Ainsi, les termes religion, sacré, numineux, rite et mythe seront employés pour mettre en jeu une spiritualité du loisir.

6. Nous comprenons le religieux «comme une mise en forme du sacré et le sacré comme matière première de la religion ». Le sacré est donc l'essence même de la religion. Voir Danièle Hervieu-Léger (1993). La religion pour mémoire, Paris, Cerf, p. 70.

7. Par «métamorphose», nous entendons une modification de la forme du sacré; elle apparaît donc dans un mouvement de déplacement et de migration dans le monde.

8. Durkheim définit la religion comme « un système solidaire de croyances et de pratiques relatives à des choses sacrées, c'est-à-dire séparées, interdites, croyances et pratiques qui unissent en une même communauté morale, appelée Église, tous ceux qui y adhèrent ». Voir Émile Durkheim (1912, p. 65)..

9. Afin de plaire aux dieux de la Grèce ancienne, les Jeux olympiques qui duraient cinq jours étaient organisés en l'honneur de Zeus. Les guerres entre les cités s'arrêtaient afin que les prêtres, les concurrents et les spectateurs puissent voyager jusqu'à l'Olympe.

10. On retrouve également la création de multiples idoles dans le monde artistique du théâtre, de la musique, de la littérature, de l'humour, etc.

11. «Pour Platon, cette identité du jeu et de l'action sacrée était reconnue sans réserve. Il n'hésitait pas à englober les choses saintes dans la catégorie du jeu. Il faut, dit-il, traiter sérieusement ce qui est sérieux, et c'est Dieu qui est digne de tout le sérieux béni, tandis que l'homme est fait pour être un jouet de Dieu, et c'est là sa meilleure part. Aussi chacun, homme ou femme, doit passer sa vie à jouer les jeux les plus beaux conformément à ce principe, et au rebours de son inclination actuelle. Car, poursuit-il, les hommes tiennent la guerre pour une chose sérieuse, «mais la guerre ne comporte ni jeu, ni éducation, précisément ce que nous tenons pour la chose la plus sérieuse. Chacun doit remplir le mieux possible la vie pacifique. Quelle est alors la juste manière ? Il faut vivre la vie en jouant certains jeux, sacrifices, chants et danses, pour gagner la faveur des dieux et pour pouvoir repousser les ennemis et triompher dans le combat. » (Huizinga, 1938, p. 43-44.)

12. Le mot grâce vient du latin gracia et du grec charis qui est employé dans la traduction biblique des Septantes pour exprimer l'hébreu hén. Celui-ci veut dire «la faveur témoignée à quelqu'un ».

13. Dans la tradition de l'hindouisme, il existe la représentation d'un dieu ludique de la création en la personne de Çiva, «le Bienfaisant». Il est le dieu de la danse qui exprime les cinq activités divines : création de l'univers, conservation, destruction du monde, incarnation des âmes, libération des âmes du cycle des existences. Sa danse cosmique est considérée comme le processus du changement succédant au changement. Des danses rituelles sont consacrées en son honneur. Il est représenté par une statue sous la forme du dieu dansant, le Nataraja, le « roi des danseurs ». 
14. Il est pertinent de souligner que Moltmann traduit ex nihilo par pour rien (notion de gratuité) aussi bien que par de rien (à partir du néant).

15. Nous adhérons à la définition phénoménologique de Waaijman qui soutient que «la spiritualité est la transformation continue impliquée dans la relation engagée ou engageante avec l'Inconditionnel» (cité dans Peelman, 1996, p. 33). De plus, le mot «spirituel» vient du vocabulaire biblique ruah en hébreu, pneuma en grec, et il correspond à spiritus en latin. Étymologiquement, le mot «spirituel» prend le sens de «mouvement de la respiration» ou du «souffle vital de l'âme». Dans la perspective occidentale, le spirituel renvoie aux « choses de la vie », à la «vitalité », à ce qui est «vivant» et se traduit par «l'éveil de la conscience».

\section{BIBLIOGRAPHIE}

Augé, M. (1982). Football : De l'histoire sociale à l'anthropologie religieuse, Le Débat, $\mathrm{n}^{\circ} 19$, p. 59-67.

Berger, P. (1971). La religion dans la conscience moderne. Paris : Centurion.

Bergson, H. (1919). L'énergie spirituelle. Paris : Presses universitaires de France.

Boff, L. (1985). Jésus-Christ libérateur. Paris : Cerf.

Bouet, M. (1968). Signification du sport. Paris: Presses universitaires de France.

Brohm, J.-M. (1976). Sociologie politique du sport. Paris : Delarge.

CAILloIs, R. (1950). L'homme et le sacré. Paris : Gallimard.

Coles, R. W. (1975). Football as a «Surrogate Religion?» A Sociological Yearbook of Religion in Britain. Londres, $\mathrm{n}^{\circ}$ 8, p. 61-75.

DAVy, M.-M. (1964). Nicolas Berdiaev : L'homme du huitième jour. Paris : Flammarion.

De Certeau, M. (1980). Arts de faire. Paris : Union générale.

DESCHAMPS, C. (1993). L'approche phénoménologique en recherche. Montréal : Guérin Universitaire.

Drewermann, E. (1993). Fonctionnaires de Dieu. Paris : Albin Michel.

Dubos, R. (1974). Choisir d'être humain. Paris : Denoël.

Dufour, R. (1977). Des mythes, du loisir/tourism., Thèse de doctorat, Université d'AixMarseille, Centre des Hautes Études touristiques.

Dumont, F. (1987). L'institution de la théologie: Essai sur la situation du théologien. Montréal : Fides.

DUMONT, F. (1995). Raisons communes. Louiseville : Boréal.

DURKHEIM, É. (1912). Les formes élémentaires de la vie religieuse : le système totémique en Australie. Paris : Presses Universitaires de France.

Eliade, M. (1965). Le sacré et le profane. Paris : Gallimard.

FOULQUIÉ, P. (1962). Dictionnaire de la langue philosophique. Paris : Presses Universitaires de France.

GEFFRÉ, C. (1990). Révélation et expérience historique des hommes, Laval théologique et philosophique 46(1). Québec: Presses de l'Université Laval. 
GERGEN, K. (1991). The saturated self-dilemmas of identity in contemporary life. New York, Basic Books.

Godbey, G.C. et Gooddale, T.L. (1988). The evolution of leisure, historical and philosophical perspectives. Pennsylvania, Venture Publishing.

GODBEY, G. (1997). Leisure and leisure services in the 21st century, State College, Venture Publishing.

Granier, J. (1985). Nihilisme, Encylopaedia Universalis, Paris, Corpus no 13, p. 32-35.

Heintzman, P. (2000). Leisure and spiritual well-Being relationships : A qualitative study, Loisir et Société, 23(1), p. 41-69.

HERVIEU-LÉGER, D. (1993). La religion pour mémoire. Paris : Cerf.

HuIzInga, J. (1938). Homo ludens : essai sur la fonction sociale du jeu. Paris : Gallimard.

ISAMBERT, F.-A. (1982). Le sens du sacré, fête et religion populaire. Paris : Minuit.

Kelly, J.R. (1987). Freedom to be - A New Sociology of Leisure, New York, Macmillan Publishing Company.

KELLY, J. R. (1997). Leisure as life : Outline of a poststructuralist reconstruction, Loisir et Société, 20(2), p. 401-418.

Lanfant, M.-F. (1972). Les théories du loisir. Paris : Presses Universitaires de France.

LIPOVETSKI, G. (1983). L'ère du vide : essaie sur l'individualisme contemporain. Paris : Gallimard.

MAFFESOLI, M. (1996). Éloge de la raison sensible. Paris : Grasset.

MASLOW, A. (1968). Vers une psychologie de l'être. Paris : Fayard.

MCDONALD, B.L., et SCHREYER, R. (1991). Spiritual benefits of leisure participation and leisure settings, dans B.L. Driver, P.J. Brown et G.L. Peterson (dir.), Benefits of leisure, State College, PA : Venture Publishing, p. 179-194.

Merleau-Ponty, M.(1945). Phénoménologie de la perception. Paris : Gallimard.

Moltmann, J. (1977). Le Seigneur de la danse. Paris : Cerf-Mame.

Moltmann, J. (1988). Dieu dans la création. Paris Cerf.

Moltmann, J. (1993). Jésus, le messie de Dieu. Paris Cerf.

OTTо, R. (1917). Le sacré: L'élément non rationnel dans l'idée du divin et sa relation avec le rationnel. Paris: Payot.

PARÉ, Jean-Louis (1997). L'intégration du migrant par les loisirs, Pourquoi partir ? La migration des jeunes d'hier et d'aujourd'hui. Québec : Presses de l'Université Laval, p. 193-194.

Piotte, J.-M. (1999). Les grands penseurs du monde occidental. Montréal : Fides.

PeElman, A. (1996). Spiritualité et conscience planétaire, Spiritualité contemporaine : défis culturels et théologiques dans Camil Ménard et Florent Villeneuve (dir.), Montréal : Fides, Héritage et Projet, nº 56, p. 21-53.

Pronovost, G. (1997). Loisir et société : Traité de sociologie empirique, $2^{\mathrm{e}}$ édition. Sainte-Foy: Presses Université du Québec.

SCHILlEBEECKX, E. (1968). La théologie du renouveau parle de Dieu, dans Laurence K. Shook, et Guy-M. Bertrand, (dir.), La théologie du renouveau, tome 1, Montréal, Paris : Fides, Cerf, p. 91-109.

TESSIER, R. (1991). Le sacré, Montréal, Paris : Fides, Cerf,. 
TILliCh, P. (1969). La dimension oubliée. Bruges: Desclée de Brouwer.

VolAnt, É. (1976). Le jeu des affranchis : Confrontation Marcuse-Moltmann, Montréal, Fides, Héritage et Projet, $\mathrm{n}^{\circ} 18$.

Waldenfels, H. (1990). Manuel de théologie fondamentale. Paris : Cerf.

WiLliams, R. (1998). Péché, dans Jean-Yves Lacoste, (dir.), Dictionnaire critique de théologie. Paris : Presses Universitaires de France, p. 872-875.

Wunenburger, J-J. (1981). Le sacré. Paris : Presses Universitaires de France.

Gervais DESCHÊNES

Pour une spiritualité du loisir

\title{
RÉSUMÉ
}

Cette étude particulière propose une approche interdisciplinaire pour développer une vision spirituelle du loisir. À partir du modèle théorique de John R. Kelly, nous proposons la création d'une neuvième et dixième métaphore, soit celles de la dimension du «religieux» et de la «transcendance». La neuvième métaphore s'inspire d'Émile Durkheim en sociologie et de Rudolf Otto en phénoménologie religieuse. Ils nous fournissent les points d'ancrage pour les prémisses d'un discours théorique sur la manifestation du sacré dans la pratique du loisir. La dixième métaphore est finalement celle de la «transcendance » qui est, avant tout, ludique. Cette métaphore s'appuie principalement sur les travaux théologiques de Karl Rahner et de Jürgen Moltmann.

Gervais DESCHÊNES

Giving a spiritual dimension to leisure

\begin{abstract}
This specific study proposes an interdisciplinary approach to develop a spiritual vision of leisure. Based on John R. Kelly's theoretical model, we are suggesting the creation of a ninth and a tenth metaphor to take into account the "religious" and the "transcendental" dimensions. The ninth metaphor draws its inspiration from Émile Durkheim, in sociology, and Rudolf Otto, in religious phenomenology. They provide us with the anchor points for the premises of a theoretical discourse on the manifestation of the sacred within the practice of leisure. The tenth metaphor,
\end{abstract}


finally, is that of "transcendency", which is, first and foremost, a ludic phenomenon. This metaphor has its main roots in the theological works of Karl Rahner and Jürgen Moltmann.

\section{Gervais DESCHÊNES}

Por una espiritualidad del ocio

\section{RESUMEN}

Este estudio particular propone un enfoque interdisciplinario para desarrollar una visión espiritual del ocio. A partir del modelo teórico de John R. Kelly, sugerimos la creación de una novena y décima metáfora que son las de la dimensión de lo «religioso » y de la «trascendencia». La novena metáfora se inspira de Émile Durkheim en sociología y de Rudolf Otto en fenomenología religiosa. Ellos nos proveen los puntos de fijación para las premisas de un discurso teórico sobre la manifestación de lo sagrado en la práctica del ocio. La décima metáfora es finalmente la de la «trascendencia » que se revela, ante todo, lúdica. Esta metáfora se apoya principalmente de los trabajos teológicos de Karl Rahner y de Jürgen Moltmann. 